\title{
EPÍFITOS VASCULARES SOBRE ESPÉCIMES DE FICUS ORGANENSIS ISOLADOS NO NORTE DA PLANÍCIE COSTEIRA DO RIO GRANDE DO SUL: PADRÕES DE ABUNDÂNCIA E DISTRIBUIÇÃO
}

\author{
Cezar Neubert Gonçalves ${ }^{1}$ \\ Jorge Luiz Waechter ${ }^{2}$
}

Recebido em 10/08/2001. Aceito em 26/04/2002.

\begin{abstract}
RESUMO - (Epífitos vasculares sobre espécimes de Ficus organensis isolados no norte da planície costeira do Rio Grande do Sul: padrões de abundância e distribuição). Os padrões de abundância e distribuição de epífitos vasculares foram estudados em espécimes isolados de Ficus organensis (Miq.) Miq. no norte da planície costeira do Rio Grande do Sul. A área de estudo está situada próximo ao Município de Terra de Areia (29³5'S; 5004' W), uma região com clima subtropical úmido (Cfa). Um total de 60 árvores foram escaladas para o inventário dos epífitos vasculares. A abundância relativa foi estimada para as espécies e o índice de diversidade de Shannon para a comunidade. A distribuição espacial dos epífitos vasculares foi estimada analisando sua ocorrência em segmentos estruturais das árvores hospedeiras (fuste, copas interna e externa) e aplicando uma técnica de análise multivariada (PCO). A composição florística resultou em 77 espécies, 32 gêneros e 10 famílias, com um índice de Shannon de 3,519 nats. Quatro espécies apresentaram valores de importância distintamente maiores, 12 valores intermediários e 61 valores relativamente menores. O mesmo padrão foi obtido com a ordenação das espécies. A riqueza epifítica por segmentos das árvores hospedeiras mostrou-se maior na copa interna, refletindo um hábitat mais favorável provido pelos ramos espessos e horizontais. A diversidade comunitária é relativamente alta considerando-se uma única espécie de forófito e um conjunto de ambientes perturbados. A distribuição das espécies epifíticas em três grupos em função das estimativas de abundância foi previamente observada para florestas costeiras melhor preservadas na mesma região.
\end{abstract}

Palavras-chave - Epifitismo, abundância, distribuição, figueiras, Sul do Brasil

\begin{abstract}
Vascular epiphytes on isolated specimens of Ficus organensis in the northern coastal plain of Rio Grande do Sul: abundance and distribution patterns). Abundance and distribution patterns of vascular epiphytes were studied on isolated specimens of Ficus organensis in the northern coastal plain of Rio Grande Sul. The study area lies around Terra de Areia town $\left(29^{\circ} 35^{\prime} \mathrm{S} ; 50^{\circ} 04^{\prime} \mathrm{W}\right)$, a region with a humid subtropical climate (Cfa). A total of 60 trees were climbed for the inventory of vascular epiphytes. Abundance parameters were estimated for the species and the Shannon diversity index was calculated for the community. Spatial distribution of vascular epiphytes was detected in two ways, one analyzing occurrence on structural segments of host trees (stem, inner and outer crown) and other applying a multivariate technique (principal coordinate analysis). Floristic composition resulted in 77 species, 32 genera and 10 families. The Shannon index of diversity resulted in 3.519 nats/individual. Four species presented a distinct higher value of importance, 12 species presented intermediate values and 61 relatively lower values. The same pattern was obtained with the ordination of species. Epiphytic richness according to host tree segments showed the higher species number in the inner crown. The community diversity may be considered as relatively high for a study with a single phorophyte species and a set of disturbed environments. The distribution of
\end{abstract}

\footnotetext{
${ }^{1}$ Biologo, Doutorando em Botanica, UFRGS, Bolsista CNPQ

${ }^{2}$ Professor do PPG - Botanica/UFRGS
} 
epiphytic species into three distinct groups according to abundance estimates was previously observed for wellpreserved coastal plain forests of the same region. The highest richness in the inner crown obviously reflects a suitable habitat provided by relatively thick and horizontal branches.

Key words - Epiphytism, abundance, distribution, fig tree, Southern Brazil

\section{Introdução}

A estrutura comunitária do componente epifítico vascular tem sido alvo de diferentes abordagens nos neotrópicos, onde o número elevado de espécies freqüentemente dificulta a realização de levantamentos fitossociológicos (Waechter 1992; Dislich \& Mantovani 1998). Como consequência, diversos autores trabalharam apenas com um ou alguns grupos taxonômicos, como Orchidaceae (Catling et al. 1986; Tremblay 1997; Nunes \& Waechter 1998; Tremblay et al. 1998/Zotz 1998), Bromeliaceae (Fontoura 1995; Zotz \& Andrade 1998; Mondragón et al. 1999), Araceae (Mantovani 1999), Cactaceae (Andrade \& Nobel 1997), e pteridófitas (Madison 1979; Zapfack et al. 1996; Hooper \& Haufler 1997; Senna \& Waechter 1997; Labiak \& Prado 1998), entre outros. Os trabalhos podem enfocar ainda uma categoria ecológica, como os hemiepífitos (Daniels \& Lawton 1993; Prosperi et al. 1995; Putz et al. 1995). Áreas de vegetação secundária, que normalmente possuem um menor número de espécies, permitem que se efetuem estudos com maior detalhamento (Dislish \& Mantovani 1998), o mesmo acontecendo em regiões subtropicais e temperadas (Brown 1990; Dickinson et al. 1993; Waechter 1992, 1998b; Jarman \& Kantvilas 1995; Dittrich et al. 1998; Campbell et al. 1999; Sillett 1999; Kersten \& Silva 2001).

A distribuição dos epífitos vasculares nos seus forófitos foi abordada em um estudo clássico por Johansson (1974), onde as árvores foram divididas verticalmente em cinco zonas. Waechter (1992) utilizou a divisão em intervalos de três metros de altura cada para verificar a distribuição de todas as espécies de epífitos vasculares em Torres e no Taim (RS). Tanto a divisão das árvores em zonas como em intervalos regulares de altura têm sido aplicadas por diversos autores (Bøgh 1992; Zimmerman \& Olmsted 1992; Hietz \& Hietz-Seifert 1995b; Freiberg 1996; Mursidawati et al. 1999; Kersten \& Silva 2001).

A maioria dos trabalhos enfocando epífitos em árvores isoladas costuma listar as espécies, não discutindo aspectos fitossociológicos (Willians-Linera et al. 1995; Guevara et al. 1998; Otero-Anaiz et al. 1999). Em pelo menos um destes levantamentos (Willians-Linera et al. 1995) foi possível demonstrar a ocorrência, nas árvores isoladas, de espécies de orquidáceas não encontradas em remanescentes florestais e plantações de café da mesma região.

No Brasil, o epifítismo vascular foi abordado em poucos trabalhos, que foram realizados em áreas florestais (Aguiar et al. 1981; Waechter 1986, 1992, 1998b; Fontoura et al. 1997; Dislich \& Mantovani 1998; Nunes \& Waechter 1998; Dittrich et al. 1999; Kersten \& Silva 2001) ou savanas (Braga \& Braga 1975; Gottsberger \& Morawetz 1993). Os padrões de abundância e a distribuição vertical das espécies epifíticas foram analisados em apenas alguns destes trabalhos (Aguiar et al. 1981; Waechter 1992, 1998b; Kersten \& Silva 2001). Entretanto, não há, para o Brasil, referência alguma à situação da flora epifítica vascular que ocorre em árvores isoladas em áreas abertas naturais ou originadas pela ação antrópica. No litoral do Rio Grande do Sul é comum a ocorrência de árvores nesta situação, principalmente figueiras, que têm uma grande importância fisionômica na paisagem local (Carauta 1989; Mello-Filho \& Neves 1989; 
Rambo 1994) e podem contribuir para manter a diversidade da sinúsia epifítica.

O presente trabalho tem por objetivo analisar os padrões de abundância e a distribuição espacial, nos fustes e nas copas das árvores, dos epífitos vasculares presentes em espécimes isolados de figueiras da espécie Ficus organensis (Miq.) Miq. do norte da planície litorânea do estado do Rio Grande do Sul, Brasil.

\section{Material e métodos}

Área de estudo - localiza-se nos municípios de Terra de Areia e Capão da Canoa (29 $33^{\circ}$ à $29^{\circ}$ $39^{\prime} \mathrm{S}$ e $50^{\circ} 00^{\prime}$ à $50^{\circ} 05^{\prime} \mathrm{W}$ ), na planície litorânea do Rio Grande do Sul. O clima na região é do tipo Cfa de Koeppen (Nimer 1979), com temperaturas médias anuais em torno de $19{ }^{\circ} \mathrm{C}$. A pluviosidade na estação meteorológica mais próxima (Maquiné) situa-se em torno de 1600 mm anuais (Waechter 1992; Sevegnani \& Baptista 1996). Em outras estações próximas, a pluviosidade varia de $1277 \mathrm{~mm}$, em Torres, à 1326 mm em Imbé (Waechter 1992, 1998a). Os solos variam de cambissolos, na proximidade da encosta do Planalto das Araucárias (Serra Geral), a planossolos, na região mais próxima ao Oceano Atlântico (IBGE 1986). O relevo na planície é varia de plano a suavemente ondulado, com altitudes variando em torno de $20 \mathrm{~m}$. Na Serra Geral, próximo à área de estudo, a altitude chega à 948 m (Ministério do Exército 1979). A vegetação local encontra-se bastante alterada pela ação antrópica, havendo alguns fragmentos de florestas arenosas, turfosas e pluviais (C. N. Gonçalves, com. pess.).

Métodos de coleta e análise - a amostragem foi realizada no período de maio de 1998 a julho de 1999. A espécie de forófito escolhida para este estudo foi Ficus organensis Miq. (Miq.), devido à sua importância fisionômica no Rio Grande do Sul (Carauta 1989; Mello-Filho \& Neves 1989). Foram consideradas como "figueiras isoladas" aquelas que não fizessem parte do dossel de uma floresta, mesmo que possuíssem uma outra árvore ocorrendo próximo (i. e., com as copas tocando-se), ou que estivessem agrupadas, formando um "parque". Como há três tipos de formações florestais (pluvial, arenosa e turfosa, senso Waechter 1985) na região onde se localiza a área de estudo, além da zona urbana da cidade de Terra de Areia, a seleção das árvores a serem pesquisadas foi feita de modo a obter-se o mesmo número de árvores (15) próximas a cada ambiente, totalizando 60 figueiras amostradas, com perímetro (ou soma de perímetros) a 1,30 m (PAP) igual ou superior a $30 \mathrm{~cm}$.

As árvores foram escaladas e os epífitos vasculares encontrados registrados, determinando-se as frequências absoluta e relativa $\left(\mathrm{Fa}_{\mathrm{i}}, \mathrm{Fr}_{\mathrm{i}}\right)$, conforme Waechter (1992, 1998b). A cobertura $\left(C_{i}\right)$ de cada espécie sobre as figueiras foi estimada considerando-se todo o forófito e utilizando-se a seguinte escala: 1 (0-15\%), 2 (15-30 $\%), 3(30-45 \%), 4(45-60 \%), 5(60-75 \%)$ e 6 (75-100\%). Os valores foram convertidos para o valor médio percentual da classe, calculandose as coberturas absoluta e relativa $\left(\mathrm{Ca}_{\mathrm{i}}, \mathrm{Cr}_{\mathrm{i}}\right)$. A partir do último índice e da frequência relativa calculou-se o valor de importância das espécies epifíticas $\left(\mathrm{VI}_{\mathrm{e}}=\mathrm{Cr}_{\mathrm{i}}+\mathrm{Fr}_{\mathrm{i}} / 2\right)$. $\mathrm{O}$ índice de Shannon $\left(\mathrm{H}^{\prime}\right)$ foi estimado, conforme Waechter (1998b), para a comparação com outros levantamentos da sinúsia epifítica vascular, realizados na mesma província geomorfológica (Justus et al. 1986) e com o mesmo número (60) de forófitos (Waechter 1992, 1998b). Uma análise multivariada foi realizada tendo o índice de similaridade de Jaccard ( $\mathrm{Ij}$ ) como medida de semelhança e a análise de coordenadas principais (PCO) como método de ordenação.

Foram eliminadas das matrizes as espécies com apenas um registro, para reduzir a indeterminação dos dados (Digby \& Kempton 1987; Podani 1994). O programa usado para esta análise foi o MULTIV (Pillar 1996). 
A análise da distribuição das espécies epifíticas nos forófitos foi efetuada com sua divisão em três segmentos: fuste (região entre a base da figueira e o ponto de onde partem os ramos principais - aqueles que apresentassem pelo menos $10 \%$ do PAP), copa interna (entre a inserção dos ramos principais e a metade da extensão destes) e copa externa (a metade final da extensão dos ramos). Esta metodologia é uma simplificação do sistema proposto por Johansson (1974), e foi adotada devido a pequena altura dos fustes nas figueiras e a horizontalidade de seus ramos (C. N. Gonçalves, dados não publicados), que tornam pouco prática a divisão da copa em cinco segmentos. A ocorrência dos epífitos vasculares em cada segmento foi registrada, calculando-se a média aritmética do número de espécies presentes.

\section{Resultados}

A composição florística resultou em 77 espécies, 33 gêneros e 10 famílias. Os maiores valores de importância foram de Microgramma vacciniifolia, Tillandsia usneoides, Tillandsia aeranthos e Rhipsalis teres (Tab. 1). Com altos valores de frequência e cobertura, estas espécies formaram um grupo isolado (Fig. 1), totalizando $\mathrm{VI}_{\mathrm{e}}=40,3 \%$. Outro grupo, de valores intermediários, com 12 espécies (Lepismium cruciforme, Tillandsia stricta, Vriesea gigantea, Aechmea nudicaulis, T. gardneri,

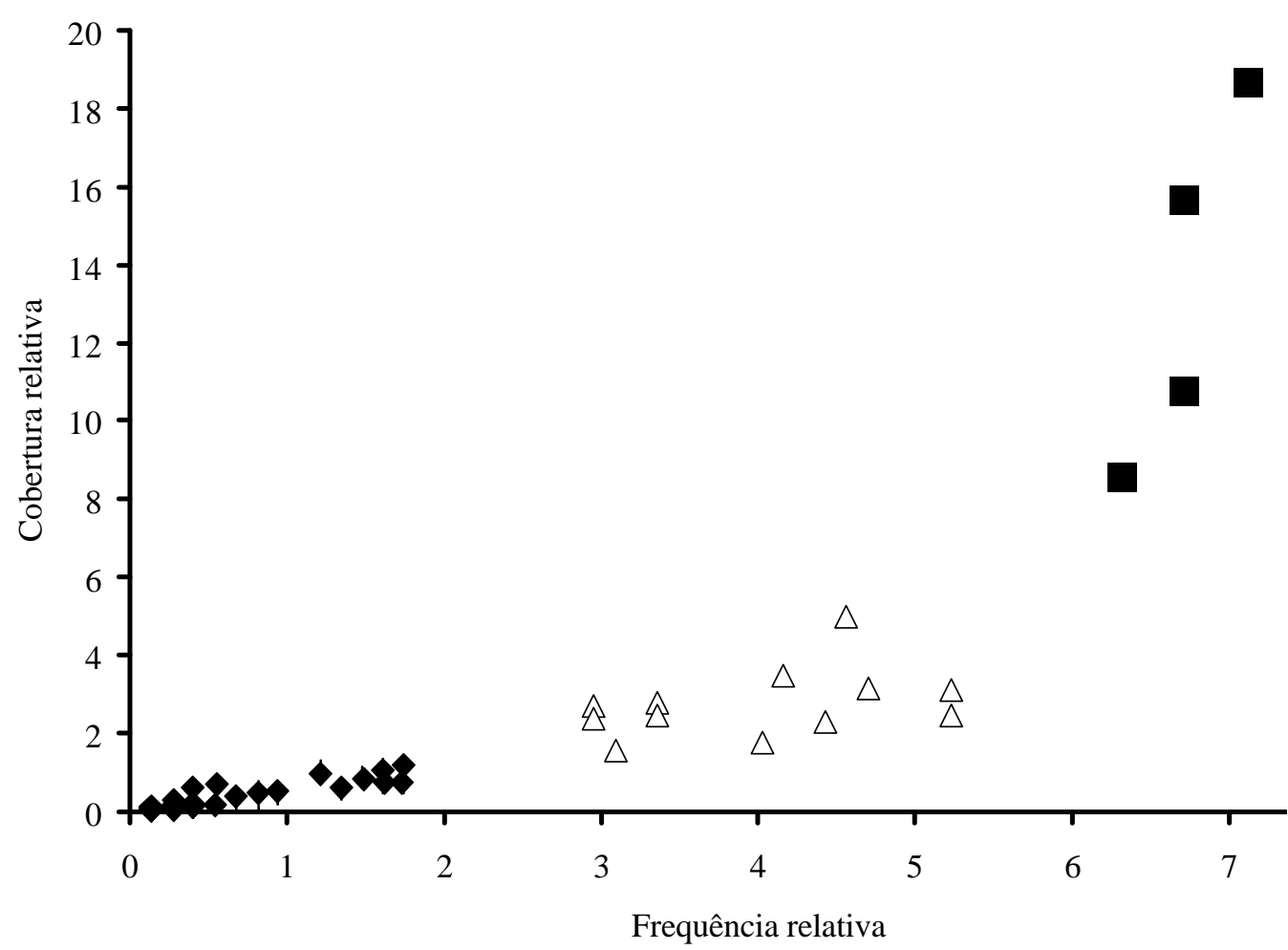

Figura 1. Relação entre frequência e cobertura relativas das espécies epifíticas. Três grupos são identificáveis: um grupo de espécies com altos valores de cobertura e frequência (pioneiras = quadrados escuros); um grupo intermediário (= triângulos claros); e outro formados pelas espécies restantes (= losangos escuros). 
Tabela 1. Estimativas de frequência e cobertura das espécies epifíticas amostradas sobre Ficus organensis isoladas no norte da planície litorânea do Rio Grande do Sul: número de indivíduos forofíticos ocupados $\left(\mathrm{Nf}_{\mathrm{i}}\right)$; Frequência absoluta $\left(\mathrm{Fa}_{\mathrm{i}}\right)$ e relativa $\left(\mathrm{Fr}_{\mathrm{i}}\right)$ sobre indivíduos forofíticos; total das estimativas de cobertura das espécies epifíticas $\left(\mathrm{C}_{\mathrm{i}}\right)$; coberturas absoluta $\left(\mathrm{Ca}_{\mathrm{i}}\right)$ e relativa $\left(\mathrm{Cr}_{\mathrm{i}}\right)$, sobre indivíduos forofíticos, em percentuais; valor de importância das espécies epifíticas $(\mathrm{VI})$.

\begin{tabular}{|c|c|c|c|c|c|c|c|c|}
\hline Espécie & Família & $\mathrm{Nf}_{\mathrm{i}}$ & $\mathrm{Fa}_{\mathrm{i}}$ & $\mathrm{Fr}_{\mathrm{i}}$ & $\mathrm{C}_{\mathrm{i}}$ & $\mathrm{Ca}_{\mathrm{i}}$ & $\mathrm{Cr}_{\mathrm{i}}$ & $\mathrm{Vic}$ \\
\hline $\begin{array}{l}\text { Microgramma vacciniifolia (Lansd. \& Fisch) } \\
\text { Copel. }\end{array}$ & Polypodiaceae & 53 & 88.30 & 7.11 & 241 & 52.60 & 18.70 & 12.90 \\
\hline illandsia usneoides (L.) L. & Bromeliaceae & 50 & 83.30 & 6.71 & 213 & 44.40 & 15.70 & 11.20 \\
\hline Tillandsia aeranthos (Loisel.) L. B. Smith & Bromeliaceae & 50 & 83.30 & 6.71 & 162 & 30.40 & 10.80 & 8.74 \\
\hline Rhipsalis teres (Vell.) Steud. & Cactaceae & 47 & 78.30 & 6.31 & 129 & 24.20 & 8.57 & 7.44 \\
\hline Lepismium cruciforme (Vell.) Miq. & Cactaceae & 34 & 56.70 & 4.56 & 113 & 14.10 & 5.01 & 4.78 \\
\hline Tillandsia stricta Soland & Bromeliaceae & 39 & 65.00 & 5.23 & 70 & 8.75 & 3.10 & 4.17 \\
\hline Aechmea nudicaulis (L.) Griseb. & Bromeliaceae & 35 & 58.30 & 4.70 & 71 & 8.87 & 3.15 & 3.92 \\
\hline Vriesea gigantea Gaud. & Bromeliaceae & 39 & 65.00 & 5.23 & 55 & 6.87 & 2.44 & 3.83 \\
\hline Tillandsia gardneri Lindl. & Bromeliaceae & 31 & 51.70 & 4.16 & 78 & 9.75 & 3.46 & 3.81 \\
\hline Vriesea procera (Mart. ex Schult. f.) Wittm. & Bromeliaceae & 33 & 55.00 & 4.43 & 52 & 6.50 & 2.30 & 3.37 \\
\hline Anthurim scandens (Aubl.) Engl. & Araceae & 25 & 41.70 & 3.36 & 63 & 7.87 & 2.79 & 3.08 \\
\hline Tillandsia recurvata $(\mathrm{L}.) \mathrm{L}$. & Bromeliaceae & 30 & 50.00 & 4.03 & 40 & 5.00 & 1.77 & 2.90 \\
\hline Microgramma squamulosa (Kaulf.) Sota & Polypodiaceae & 25 & 41.70 & 3.36 & 55 & 6.87 & 2.44 & 2.90 \\
\hline Tillandsia geminiflora Brongn. & Bromeliaceae & 22 & 36.70 & 2.95 & 61 & 7.62 & 2.70 & 2.83 \\
\hline Brassavola tuberculata Hook. & Orchidaceae & 22 & 36.70 & 2.95 & 54 & 6.75 & 2.39 & 2.67 \\
\hline Tillandsia mallemontii Glaz. ex Mez & Bromeliaceae & 23 & 38.30 & 3.09 & 35 & 4.37 & 1.55 & 2.32 \\
\hline Octomeria crassifolia Lindl. & Orchidaceae & 13 & 21.70 & 1.74 & 25 & 3.12 & 1.11 & 1.42 \\
\hline Tillandsia tenuifolia $L$. & Bromeliaceae & 12 & 20.00 & 1.61 & 24 & 3.00 & 1.06 & 1.34 \\
\hline Pleurothallis exarticulata B. Rodr. & Orchidaceae & 13 & 21.70 & 1.74 & 17 & 2.12 & 0.75 & 1.25 \\
\hline Cattleya intermedia Grah. ex Hook. & Orchidaceae & 12 & 20.00 & 1.61 & 17 & 2.12 & 0.75 & 1.18 \\
\hline Peperomia pereiskiifolia (Jacq.) HBK. & Piperaceae & 11 & 18.30 & 1.48 & 19 & 2.37 & 0.84 & 1.16 \\
\hline Pleurothallis glumacea Lindl. & Orchidaceae & 9 & 15.00 & 1.21 & 23 & 2.87 & 1.02 & 1.11 \\
\hline Epidendrum pseudodifforme Schltr. & Orchidaceae & 10 & 16.70 & 1.34 & 13 & 1.62 & 0.57 & 0.96 \\
\hline Polypodium hirsutissimum Raddi & Polypodiaceae & 7 & 11.70 & 0.94 & 10 & 1.25 & 0.44 & 0.69 \\
\hline Codonanthe devosiana Lem. & Gesneriaceae & 6 & 10.00 & 0.81 & 11 & 1.37 & 0.49 & 0.65 \\
\hline Polypodium pleopeltifolium Raddi & Polypodiaceae & 4 & 6.70 & 0.54 & 15 & 1.87 & 0.66 & 0.60 \\
\hline Aechmea recurvata (K1.) L. B. Smith & Bromeliaceae & 6 & 10.00 & 0.81 & 7 & 0.87 & 0.31 & 0.56 \\
\hline Coussapoa microcarpa (Schott) Rizzini & Cecropiaceae & 5 & 8.30 & 0.67 & 7 & 0.87 & 0.31 & 0.49 \\
\hline Vriesea friburgensis $\mathrm{Mez}$ & Bromeliaceae & 3 & 5.00 & 0.40 & 10 & 1.25 & 0.44 & 0.42 \\
\hline Rhipsalis paradoxa Salm-Dyck & Cactaceae & 4 & 6.60 & 0.54 & 4 & 0.50 & 0.18 & 0.36 \\
\hline Aechmea gamosepala Wittm. & Bromeliaceae & 3 & 5.00 & 0.40 & 7 & 0.87 & 0.31 & 0.35 \\
\hline Rhipsalis pulvinigera Lindbg. & Cactaceae & 3 & 5.00 & 0.40 & 5 & 0.62 & 0.22 & 0.31 \\
\hline Cereus hildmannianus K. Schum. & Cactaceae & 3 & 5.00 & 0.40 & 4 & 0.50 & 0.18 & 0.29 \\
\hline Anthurium gaudichaudianum Kunth & Araceae & 3 & 5.00 & 0.40 & 3 & 0.37 & 0.13 & 0.27 \\
\hline Ficus organensis (Miq.) Miq. & Moraceae & 3 & 5.00 & 0.40 & 3 & 0.37 & 0.13 & 0.27 \\
\hline Lankesterella ceracifolia (B. Rodr.) Mansf. & Orchidaceae & 3 & 5.00 & 0.40 & 3 & 0.37 & 0.13 & 0.27 \\
\hline Pleurothallis iguapensis Schltr. & Orchidaceae & 3 & 5.00 & 0.40 & 3 & 0.37 & 0.13 & 0.27 \\
\hline Polypodium squalidum Vell. & Polypodiaceae & 2 & 3.30 & 0.27 & 6 & 0.75 & 0.27 & 0.27 \\
\hline Anthurium gaudichaudianum Kunth & Araceae & 2 & 3.30 & 0.27 & 4 & 0.50 & 0.18 & 0.22 \\
\hline Lepismium lumbricoides (Lem.) Barthl. & Cactaceae & 2 & 3.30 & 0.27 & 4 & 0.50 & 0.18 & 0.22 \\
\hline Peperomia catharinae Miq. & Piperaceae & 2 & 3.30 & 0.27 & 3 & 0.37 & 0.13 & 0.20 \\
\hline Peperomia tretraphylla (Forst.) & Piperaceae & 2 & 3.30 & 0.27 & 2 & 0.25 & 0.09 & 0.18 \\
\hline \multicolumn{9}{|l|}{ Hook. \& Arn. } \\
\hline Encyclia vespa (Vell.) Dressl. & Orchidaceae & 2 & 3.30 & 0.27 & 2 & 0.25 & 0.09 & 0.18 \\
\hline Lanium avicula (Lindl.) Hoehne & Orchidaceae & 2 & 3.30 & 0.27 & 2 & 0.25 & 0.09 & 0.18 \\
\hline Vriesea rodigasiana E. Morr. & Bromeliaceae & 2 & 3.30 & 0.27 & 2 & 0.25 & 0.09 & 0.18 \\
\hline
\end{tabular}


Tabela 1. (continuação).

\begin{tabular}{|c|c|c|c|c|c|c|c|c|}
\hline Espécie & Família & $\mathrm{Nf}_{\mathrm{i}}$ & $\mathrm{Fa}_{\mathrm{i}}$ & $\mathrm{Fr}_{\mathrm{i}}$ & $\mathrm{C}_{\mathrm{i}}$ & $\mathrm{Ca}_{\mathrm{i}}$ & $\mathrm{Cr}_{\mathrm{i}}$ & $\mathrm{Vi}_{\mathrm{e}}$ \\
\hline Philodendron bipinnatifidum Schott ex Engl. & Araceae & 2 & 3.30 & 0.27 & 1 & 0.12 & 0.04 & 0.16 \\
\hline Peperomia rotundifolia (L.) HBK. & Piperaceae & 1 & 1.67 & 0.13 & 3 & 0.37 & 0.13 & 0.13 \\
\hline Codonanthe gracilis (Mart.) Hanst. & Gesneriaceae & 1 & 1.67 & 0.13 & 2 & 0.25 & 0.09 & 0.11 \\
\hline $\begin{array}{l}\text { Sophronitis purpurata (Lindl.) } \\
\text { van den Berg \& Chase }\end{array}$ & Orchidaceae & 1 & 1.67 & 0.13 & 2 & 0.25 & 0.09 & 0.11 \\
\hline Lepismium houlletianum (Lem.) Barthl. & Cactaceae & 1 & 1.67 & 0.13 & 2 & 0.25 & 0.09 & 0.11 \\
\hline Octomeria oxychela B. Rodr. & Orchidaceae & 1 & 1.67 & 0.13 & 2 & 0.25 & 0.09 & 0.11 \\
\hline Oncidium flexuosum Sims & Orchidaceae & 1 & 1.67 & 0.13 & 2 & 0.25 & 0.09 & 0.11 \\
\hline Peperomia glabella (Sw.) A. Dietr. & Piperaceae & 1 & 1.67 & 0.13 & 2 & 0.25 & 0.09 & 0.11 \\
\hline Polystachya strellensis Rchb. f. & Orchidaceae & 1 & 1.67 & 0.13 & 2 & 0.25 & 0.09 & 0.11 \\
\hline Sinningia douglasii (Lindl.) Chaut. & Gesneriaceae & 1 & 1.67 & 0.13 & 2 & 0.25 & 0.09 & 0.11 \\
\hline Barbosella dusenii (A. Champ.) Schltr. & Orchidaceae & 1 & 1.67 & 0.13 & 1 & 0.25 & 0.09 & 0.11 \\
\hline Billbergia zebrina (Herb.) Lindl. & Bromeliaceae & 1 & 1.67 & 0.13 & 1 & 0.12 & 0.04 & 0.09 \\
\hline Capylocentrum aromaticum B. Rodr. & Orchidaceae & 1 & 1.67 & 0.13 & 1 & 0.12 & 0.04 & 0.09 \\
\hline Epidendrum latilabre Lindl. & Orchidaceae & 1 & 1.67 & 0.13 & 1 & 0.12 & 0.04 & 0.09 \\
\hline Epidendrum rigidum Jacq. & Orchidaceae & 1 & 1.67 & 0.13 & 1 & 0.12 & 0.04 & 0.09 \\
\hline Isabelia pulchella (Krzl.) Sengh. \& Teusch. & Orchidaceae & 1 & 1.67 & 0.13 & 1 & 0.12 & 0.04 & 0.09 \\
\hline Lepismium warmingianum (K. Schum.) Barthl. & Cactaceae & 1 & 1.67 & 0.13 & 1 & 0.12 & 0.04 & 0.09 \\
\hline Maxillaria cogniauxiana Hoehne & Orchidaceae & 1 & 1.67 & 0.13 & 1 & 0.12 & 0.04 & 0.09 \\
\hline Octomeria gracilis & Orchidaceae & 1 & 1.67 & 0.13 & 1 & 0.12 & 0.04 & 0.09 \\
\hline Oncidium fimbriatum Lindl. & Orchidaceae & 1 & 1.67 & 0.13 & 1 & 0.12 & 0.04 & 0.09 \\
\hline Oncidium gravesianum Rolfe & Orchidaceae & 1 & 1.67 & 0.13 & 1 & 0.12 & 0.04 & 0.09 \\
\hline Oncidium micropogon Rchb. f. & Orchidaceae & 1 & 1.67 & 0.13 & 1 & 0.12 & 0.04 & 0.09 \\
\hline Opuntia arechavaletae Speg. ex. Arechav. & Cactaceae & 1 & 1.67 & 0.13 & 1 & 0.12 & 0.04 & 0.09 \\
\hline Peperomia alata Ruiz \& Pavon & Piperaceae & 1 & 1.67 & 0.13 & 1 & 0.12 & 0.04 & 0.09 \\
\hline Pleurothallis aphtosa Lindl. & Orchidaceae & 1 & 1.67 & 0.13 & 1 & 0.12 & 0.04 & 0.09 \\
\hline Pleurothallis higrophila B. Rodr. & Orchidaceae & 1 & 1.67 & 0.13 & 1 & 0.12 & 0.04 & 0.09 \\
\hline Pleurothallis linearifolia Cogn. & Orchidaceae & 1 & 1.67 & 0.13 & 1 & 0.12 & 0.04 & 0.09 \\
\hline Pleurothallis cf. marginalis $\mathrm{Rchb}$. $\mathrm{f}$. & Orchidaceae & 1 & 1.67 & 0.13 & 1 & 0.12 & 0.04 & 0.09 \\
\hline Pleurothallis sarracenia Luer & Orchidaceae & 1 & 1.67 & 0.13 & 1 & 0.12 & 0.04 & 0.09 \\
\hline Rumohra adiantiformis (Forst.) Ching & Dryopteridaceae & 1 & 1.67 & 0.13 & 1 & 0.12 & 0.04 & 0.09 \\
\hline Tillandsia tricholepis Backer & Bromeliaceae & 1 & 1.67 & 0.13 & 1 & 0.12 & 0.04 & 0.09 \\
\hline Vriesea philippocoburgii Wawra & Bromeliaceae & 1 & 1.67 & 0.13 & 1 & 0.12 & 0.04 & 0.09 \\
\hline Vriesea vagans $\mathrm{L}$. B. Smith & Bromeliaceae & 1 & 1.67 & 0.13 & 1 & 0.12 & 0.04 & 0.09 \\
\hline
\end{tabular}

$V$. procera, $T$. recurvata, Anthurium scandens, Microgramma squamulosa, $T$. geminiflora, Brassavola tuberculata e $T$. mallemontii), apresentou $\mathrm{VI}_{\mathrm{e}}=40,6 \%$. Um último grupo, composto por todas as demais espécies (61), com valores de frequência e cobertura muito menores, apresentou $\mathrm{VI}_{\mathrm{e}}=$ $19,1 \%$. Embora contribuindo menos da metade dos valores de importância de cada um dos dois grupos referidos acima, este grupo corresponde a 79,22\% do total de espécies encontrado.
$\mathrm{Na}$ análise multivariada, utilizando dados de 46 espécies, foi possível identificar o mesmo padrão: as espécies distribuíram-se de acordo com as suas frequências, que aumentam da direita para a esquerda do gráfico, formando grupos que coincidem com os descritos no parágrafo anterior (Fig. 2). Assim, há um grupo de espécies presentes na maioria das árvores, mesmos nas menores, comportando-se como pioneiras, à esquerda do gráfico. Outro grupo tem frequências intermediárias e instala-se após as primeiras. À direita, ficam as espécies mais 


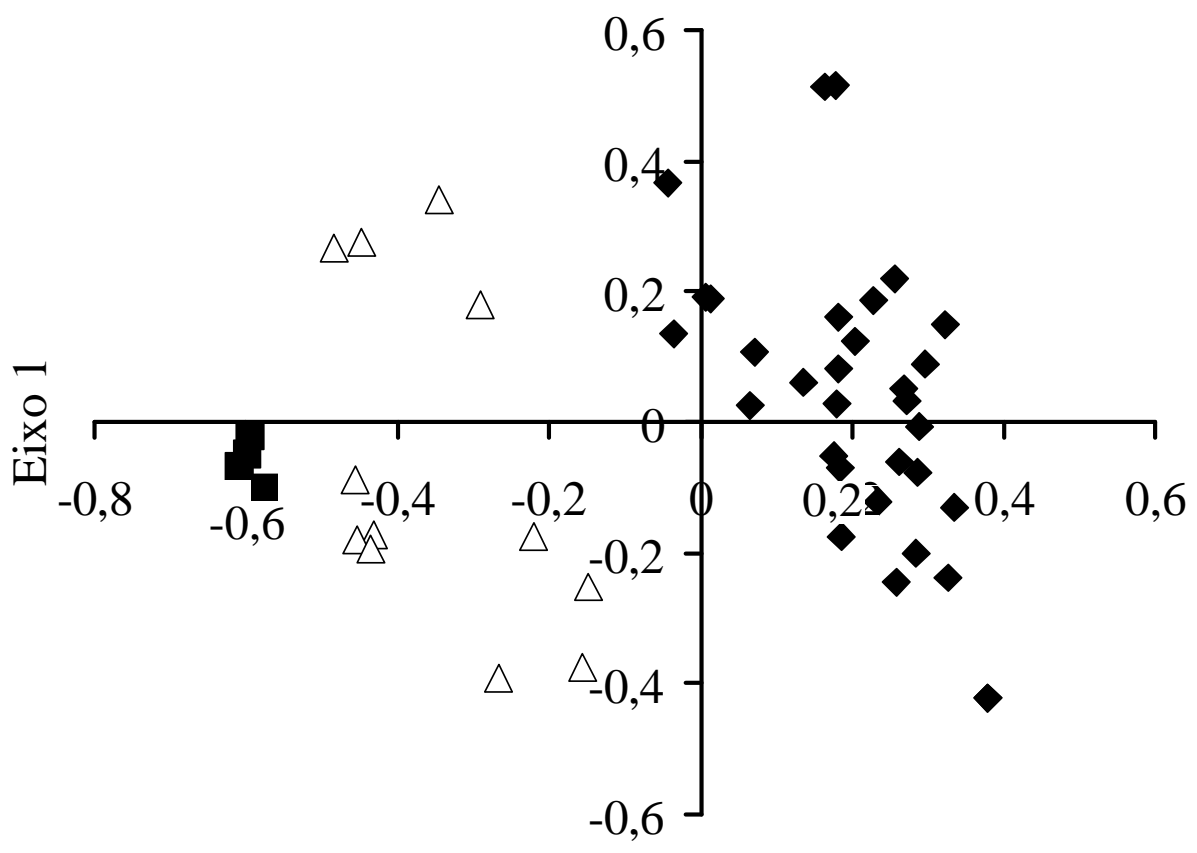

Eixo 3

Figura 2. Diagrama de ordenação das espécies epifíticas no espaço bidimensional do primeiro $(11,76 \%)$ e terceiro $(5,88 \%)$ eixos obtidos pela análise de coordenadas principais $(\mathrm{PCO})$. Quadrados escuros = pioneiras; triângulos claros = intermediárias; losangos escuros = climácicas. Os grupos correspondem aos da figura 2.

Tabela 2. Número de espécies de epífitas vasculares e índice de Shannon em levantamentos fitossociológicos efetuados na planície costeira do Rio Grande do Sul. Nf = número de forófitos; $\mathrm{N}=$ número de espécies epifíticas encontadas; H' = Indice de Shannon

\begin{tabular}{llccc}
\hline Localidade & Referência & $\mathrm{Nf}$ & $\mathrm{N}$ & $\mathrm{H}^{\prime}$ \\
\hline $\begin{array}{l}\text { Figueiras isoladas, } \\
\text { Terra de Areia }\end{array}$ & Este estudo & 60 & 77 & 3,519 \\
$\begin{array}{l}\text { Torres } \\
\begin{array}{l}\text { Estação Ecológica } \\
\text { do Taim }\end{array}\end{array}$ & Waechter 1992 & 60 & 93 & 4,049 \\
$\begin{array}{l}\text { Restinga da } \\
\text { Emboaba }\end{array}$ & Waechter 1992 & 60 & 24 & 2,886 \\
\hline
\end{tabular}

raras, que surgem quando as árvores são maiores e mais velhas (espécies climácicas).

O valor do índice de Shannon encontrado para as figueiras isoladas, 3,519 (Tab. 2), é muito mais alto que os encontrados para uma floresta turfosa do extremo sul do estado (Taim) e para uma floresta de restinga, em Osório, mas é inferior ao das matas turfosas de Torres.

Os padrões de distribuição dos epífitos nos forófitos (Fig. 3, Tab. 3) incluem espécies que, predominantemente, ocorrem: na região basal da árvore (seis espécies), ou seja, nos fustes e na copa interna, como por exemplo Peperomia pereskifolia; na copa interna (10 espécies), como em Microgramma squamulosa; na copa externa (12 espécies), como em Vriesea procera; e na copa (18 espécies), sem uma definição clara do segmento predominante, como em Tillandsia aeranthos. Não foi possível determinar o padrão de 30 espécies, presentes em apenas um forófito cada, porém a maioria 
Tabela 3. Padrões de distribuição e número de registros, em cada segmento nos quais as árvores foram divididas, das espécies epifíticas vasculares. Nfi = número de forófitos individuais ocupados pela espécie i; FUS = fuste; CIN = copa interna; CEX = copa externa. (Acrônimos formados pelas letras iniciais dos nomes das espécies, conforme tabela 1.)

\begin{tabular}{|c|c|c|c|c|c|c|c|c|c|c|c|}
\hline Padrão ${ }^{1}$ & Epífitos & Nfi & FUS & $\mathrm{CIN}$ & CEX & Padrão $^{1}$ & Epífitos & Nfi & FUS & $\mathrm{CIN}$ & CEX \\
\hline \multirow[t]{6}{*}{ Basal } & Cosmic & 5 & 4 & 4 & 0 & \multirow[t]{8}{*}{ C.indef. } & Pleigu & 3 & 0 & 1 & 2 \\
\hline & Ficorg & 3 & 1 & 2 & 0 & & Plesar & 1 & 0 & 0 & 1 \\
\hline & Pepcat & 2 & 2 & 0 & 0 & & Rhipar & 3 & 0 & 2 & 3 \\
\hline & Pepper & 9 & 9 & 9 & 1 & & Rhipul & 3 & 1 & 2 & 1 \\
\hline & Phibip & 2 & 2 & 0 & 0 & & Tilaer & 50 & 23 & 43 & 42 \\
\hline & Plosqu & 3 & 2 & 2 & 0 & & Tilrec & 25 & 9 & 18 & 19 \\
\hline \multirow[t]{10}{*}{ C.interna } & Aecgam & 4 & 1 & 3 & 1 & & Vrifri & 3 & 0 & 2 & 3 \\
\hline & Bratub & 22 & 8 & 18 & 16 & & Vrirod & 2 & 0 & 1 & 1 \\
\hline & Encves & 2 & 0 & 2 & 0 & \multirow[t]{31}{*}{ N.det. } & Peprot & 1 & 0 & 1 & 1 \\
\hline & Lepcru & 34 & 14 & 30 & 26 & & Pepala & 1 & 1 & 0 & 0 \\
\hline & Micsqu & 22 & 10 & 19 & 14 & & Plehig & 1 & 0 & 0 & 1 \\
\hline & Micvac & 53 & 40 & 50 & 46 & & Plemal & 1 & 0 & 1 & 0 \\
\hline & Polhir & 13 & 2 & 9 & 4 & & Pleaph & 1 & 0 & 0 & 1 \\
\hline & Polple & 6 & 0 & 6 & 1 & & Plemar & 1 & 0 & 1 & 0 \\
\hline & Tilmal & 23 & 3 & 15 & 13 & & Polstr & 1 & 0 & 1 & 1 \\
\hline & Tilten & 12 & 0 & 9 & 7 & & Rumadi & 1 & 0 & 1 & 0 \\
\hline \multirow[t]{12}{*}{ C.externa } & Aecnud & 31 & 3 & 21 & 27 & & Sindou & 1 & 1 & 0 & 0 \\
\hline & Antsca & 25 & 3 & 16 & 20 & & Tiltri & 1 & 1 & 0 & 0 \\
\hline & Catint & 11 & 2 & 4 & 8 & & Vriphi & 1 & 0 & 1 & 0 \\
\hline & Epidif & 7 & 0 & 3 & 5 & & Vrivag & 1 & 0 & 0 & 1 \\
\hline & Pleglu & 10 & 0 & 3 & 8 & & Baraus & 1 & 0 & 1 & 0 \\
\hline & Rhiter & 47 & 14 & 34 & 37 & & Bilzeb & 1 & 1 & 0 & 0 \\
\hline & Tilgar & 39 & 0 & 17 & 34 & & Camaro & 1 & 0 & 1 & 0 \\
\hline & Tilgem & 30 & 1 & 14 & 21 & & Codgra & 1 & 0 & 1 & 0 \\
\hline & Tilstr & 39 & 5 & 26 & 28 & & Epilat & 1 & 0 & 1 & 0 \\
\hline & Tilusn & 50 & 21 & 46 & 50 & & Epirig & 1 & 0 & 0 & 1 \\
\hline & Vrigig & 35 & 1 & 18 & 29 & & Isapul & 1 & 0 & 0 & 1 \\
\hline & Vripro & 33 & 2 & 9 & 32 & & Laepur & 1 & 1 & 1 & 0 \\
\hline \multirow[t]{11}{*}{ C.indef. } & Aecrec & 4 & 2 & 4 & 3 & & Lephou & 1 & 1 & 1 & 0 \\
\hline & Antgau & 2 & 0 & 2 & 2 & & Lepwar & 1 & 0 & 1 & 0 \\
\hline & Cerhil & 3 & 1 & 1 & 2 & & Maxcog & 1 & 0 & 1 & 0 \\
\hline & Coddev & 6 & 1 & 4 & 4 & & Octgra & 1 & 0 & 1 & 0 \\
\hline & Lanavi & 2 & 0 & 1 & 1 & & Octoxy & 1 & 0 & 1 & 1 \\
\hline & Lancer & 3 & 0 & 1 & 2 & & Oncgra & 1 & 0 & 1 & 0 \\
\hline & Leplum & 2 & 1 & 1 & 2 & & Oncfim & 1 & 1 & 0 & 0 \\
\hline & Octcra & 13 & 2 & 10 & 9 & & Oncfle & 1 & 1 & 1 & 0 \\
\hline & Peptet & 2 & 0 & 1 & 2 & & Oncmic & 1 & 0 & 1 & 0 \\
\hline & Pleexa & 12 & 2 & 6 & 7 & & Opuare & 1 & 0 & 1 & 0 \\
\hline & & & & & & & Pepgla & 1 & 1 & 1 & 0 \\
\hline
\end{tabular}

1 - Padrões: Basal = ocorre predominantemente no fuste e copa interna; C.interna = ocorre predominantemente na copa interna da árvore; C. externa = ocorre predominantemente na copa externa da árvore; $\mathrm{C}$. indef. $=$ predominante na copa, sem definição de preferência por um segmento; N. det. = não determinado. 


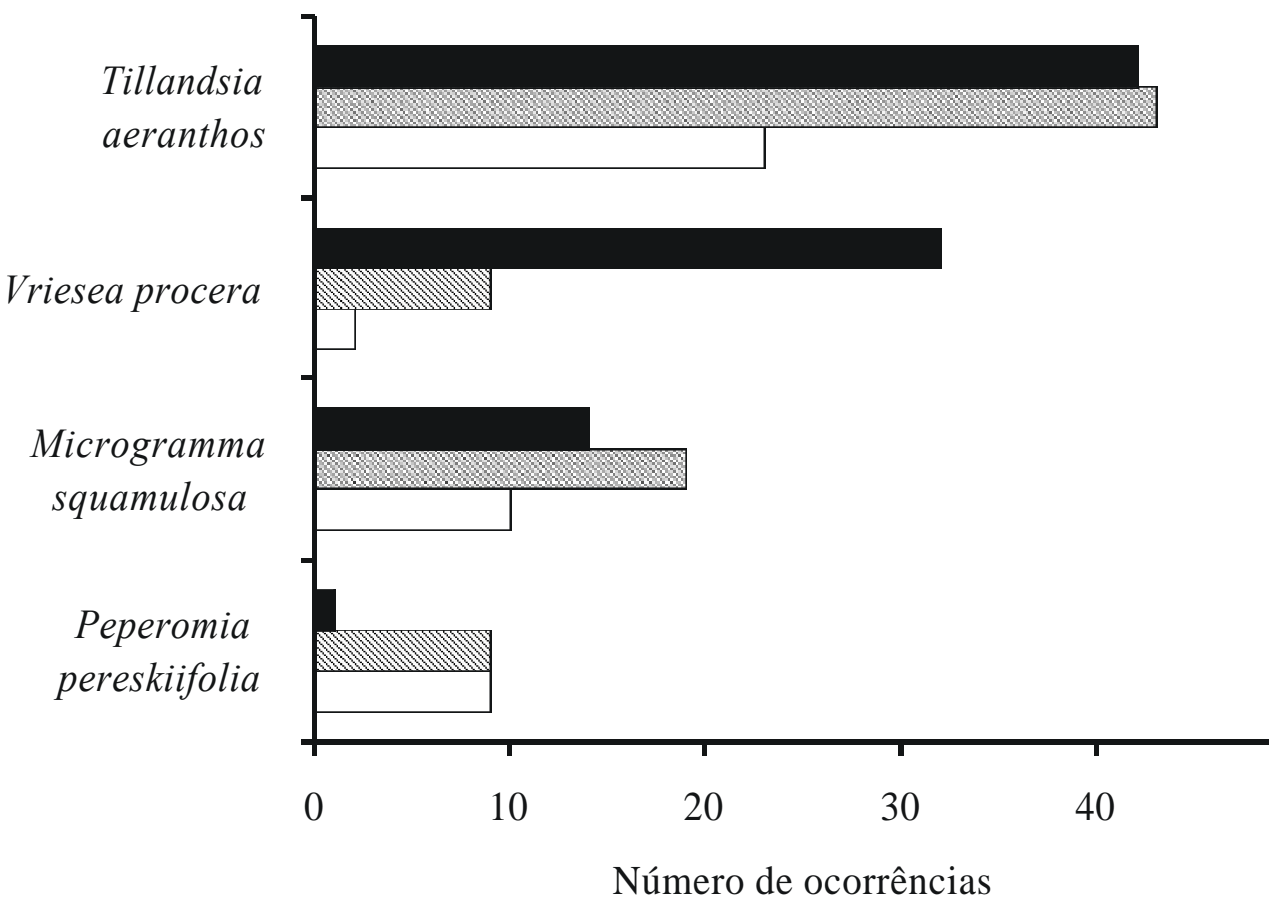

Figura 3. Exemplos dos padrões de distribuição das espécies epifíticas (segmentos dos forófitos: coluna vazada = fuste; coluna hachureada $=$ copa interna; coluna preta $=$ copa externa).

delas estava localizada na copa interna, próximas ao ponto de inserção dos ramos nos fustes. Foram encontradas 40 espécies nos fustes, 64 na copa interna e 48 na copa externa. A média aritmética do número de espécies presentes nos fustes foi de $3,35 \pm 2,25$. Na copa interna, a média foi de 8,51 $\pm 4,11$ espécies, com um grande número de espécies raras, encontradas apenas em uma árvore. Finalmente, na copa externa, a média foi de $9,03 \pm 4,11$ espécies.

\section{Discussão}

A predominância das quatro espécies pioneiras, com os maiores valores de importância, pode ser creditada a uma série de fatores, como a produção de uma grande quantidade de diásporos e a resistência às condições de maior insolação encontradas em árvores menores, conferindo-lhes a capacidade de colonizar rapidamente os forófitos jovens. Estas espécies mantêm-se como dominantes na comunidade mesmo nas árvores maiores. A ocorrência de poucas espécies com grandes valores de importância e de muitas com valores baixos espécies também foi registrada em outros levantamentos da flora epifítica vascular na região litorânea do sul do Brasil (Waechter 1992, 1998b; Kersten \& Silva 2001). Em Microgramma vacciniifolia, o rizoma reptante estende-se sobre os fustes e ramos, permitindo a ocupação de extensas áreas das copas das árvores (Dislich \& Mantovani 1998; Waechter 1998b; Kersten \& Silva 2001). Tillandsia aeranthos e T. usneoides são espécies heliófilas e que apresentam uma intensa reprodução clonal, principalmente a última espécie (Reitz 1983). Rhipsalis teres é a única espécie pioneira com 
dispersão zoocórica. É provável que a dispersão de seus frutos seja efetuada pela avifauna (A. Kindel, Depto. Ecologia UFRGS, com. pess.).

Desta forma, esta espécie pode se constituir em um importante recurso para a manutenção das aves, principalmente sobre árvores isoladas em áreas urbanas ou em campos submetidos a pastejo intenso nas quais ela ocorra. As espécies com frequências intermediárias colonizam principalmente árvores de porte maior, embora ocasionalmente possam ser encontradas em forófitos menores. Este grupo é composto tanto por espécies heliófilas, que ocorrem principalmente na copa externa (como Aechmea nudicaulis, Anthurium scandens, Tillandsia gardneri, T. geminiflora, T. stricta, Vriesea gigantea e $V$. procera), quanto por espécies que ocorrem nos ramos mais espessos, na copa interna (Brassavola tuberculata, Lepismium cruciforme, Microgramma squamulosa e Tillandsia mallemontii). É possível que estas últimas espécies sejam mais exigentes quanto às condições de substrato. Tillandsia recurvata, que ocorre preferencialmente na copa, mas sem uma definição de preferência por um segmento, pode comportar-se como espécie pioneira, tendo sido a única espécie encontrada na menor árvore amostrada. O grupo de espécies com os valores de importância menores, que representa a maior parte da diversidade biológica da sinúsia epifítica sobre as figueiras analisadas, pode ser caracterizado como climácico porque maioria das espécies só foi encontrada em árvores de grande porte, colonizando-as após as espécies dos dois grupos anteriores terem se instalado.

Os resultados obtidos na análise da distribuição espacial dos epífitos vasculares nas figueiras estão de acordo com os resultados obtidos por outros autores (Johansson 1974; Bøgh 1992; Hietz \& Hietz-Seifert 1995a; Freiberg 1996; Rudolph et al. 1998; Nieder et al. 1999), embora as metodologias utilizadas tenham sido diferentes. Os fustes são, normalmente, áreas que não favorecem a instalação de epífitos vasculares, devido principalmente à verticalidade do substrato (Bøgh 1992; Waechter 1992; Freiberg 1996), à ação do vento, à presença de animais (em especial, gado bovino) nas imediações das árvores e à pequena altura deste segmento nas árvores isoladas, mesmo considerando a presença, nas figueiras, de extensas raízes tabulares, o que aumenta a área disponível para a colonização pelos epífitos vasculares. O grande número de espécies na copa interna, por sua vez, provavelmente resulta da conjunção de uma série de fatores, como a grande espessura e a horizontalidade dos ramos, a menor incidência de radiação solar direta e a antiguidade do substrato (Waechter 1992). Os hemiepífitos e os holoepífitos acidentais e facultativos ocorrem predominantemente neste segmento, em geral em forquilhas e nos pontos onde os ramos se inserem nos fustes. No último segmento, a copa externa, há um predomínio de espécies heliófilas, especialmente bromeliáceas dos gêneros Tillandsia ( $T$. gardneri, T. geminiflora) e Vriesea ( $V$. gigantea, $V$. procera), que se desenvolvem nos ramos mais apicais. A última espécie é 3,5 vezes mais frequente na copa externa que na copa interna, e suas inflorescências podem ser facilmente avistadas emergindo acima das copas da figueiras. Já a presença preponderante de $V$. gigantea está de acordo com o padrão encontrado para esta espécie em Santa Catarina, ocorrendo principalmente nas porções terminais dos ramos, apesar do grande porte dos seus indivíduos (Reitz 1983). Além disto, as espécies que apresentam caules reptantes (Microgramma vacciniifolia, M. squamulosa, Pleurothallis exarticulata, entre outras) normalmente colonizam os ramos na maior parte da sua extensão, só não recobrindo os seus extremos apicais, sendo registradas em ambos os segmentos da copa. Desta forma, as espécies encontradas na copa externa são mais frequentes, o que explica o porque do número 
médio de espécies encontrado neste segmento foi maior em relação à copa interna.

O valor do índice de Shannon encontrado no presente estudo pode ser considerado relativamente alto, refletindo o elevado número de espécies (77). Esta diversidade pode ser condicionada pelo grande porte das árvores, pela horizontalidade de seus ramos, e pela posição setentrional, em relação a planície costeira do Rio Grande do Sul, da área de estudo.

Os dados obtidos neste trabalho mostram que as figueiras isoladas apresentam uma situação favorável ao epifitismo, mesmo considerando condições ambientais mais extremas a que os epífitos podem estar submetidos, como maior luminosidade, maior incidência de ventos e a ação do ser humano e de animais domésticos. Sua preservação pode garantir a manutenção de uma parcela importante da flora epifítica regional.

\section{Agradecimentos}

Aos funcionários do Herbário ICN, aos professores do PPG Botânica da UFRGS e aos revisores, cujas sugestões muito contribuíram para a melhoria do original. $\mathrm{O}$ primeiro autor agradece a Cristiane F. de Azevêdo-Gonçalves pelo auxílio nos trabalhos de campo, a Andreas Kindel pelas informações sobre Rhipsalis teres, e ao CNPQ pela bolsa concedida.

\section{Referências bibliográficas}

Aguiar, L. W.; Citadini-Zanette, V.; Martau, L. \& Backes, A. 1981. Composição florística de epífitos vasculares numa área localizada nos municípios de Montenegro e Triunfo, Rio Grande do Sul, Brasil. Iheringia, Série Botânica 28: 55-93.

Andrade, J. L. \& Nobel, P. S. 1997. Microhabitats and water relations of epiphytic cacti and ferns in a lowland neotropical forest. Biotropica 29(3): 261-270.

Braga, M. M. N. \& Braga, P. I. S. 1975. Estudos sobre a vegetação das campinas amazônicas IV. Ocorrência de algumas epífitas na campina e campinarana. Acta Amazônica 5(3): 254-260.

Bøgh, A. 1992. The composition and distribution of the vascular epiphyte flora of an Ecuadorian montane rain forest. Selbyana 13: 25-34.

Brown, A. D. 1990. Epyphitism in the montane forests of El Rey National Park in Argentina - Floristic composicion and distribution pattern. Revista Biología Tropical 38(2): 155-166.

Campbell, J., Stevenson, S. K., \& Coxson, D. S. 1999. Estimating epiphyte abundance in high-elevation forests of northern British Columbia. Selbyana 20(2): 261-267.

Carauta, J. P. P. 1989. Ficus (Moraceae) no Brasil: conservação e taxonomia. Albertoa 2: 1-365.

Catling, P. M., Brownnell, V. R. \& Lekovitch, L. P. 1986 Epiphytic orchids in a Belizean grapefruit orchard: distribution, colonization and association. Lindleyana 1: 194-202.

Daniels, J. D. \& Lawton, R. O. 1993. A natural history of strangling by Ficus crassiuscula in a Costa Rican lower montane rain forest. Selbyana 14: 59-63.

Dickinson, K. J. M., Mark, A. F. \& Dawkins, B. 1993. Ecology of lianoid epiphytic communities in coastal podocarp rain-forest, Haast Ecological District, New Zealand. Journal of Biogeography 20(6): 687-705.

Digby, P. G. N. \& Kempton, R. A. 1987. Multivariate analysis of ecological communities. Population and community biology series. $1^{\mathrm{a}}$ Ed. Chapman \& Hall, London, Inglaterra. 206 p. Ilustr.

Dislich, R. \& Mantovani, W. 1998. A flora de epífitas da reserva da Cidade Universitária "Armando de Salles Oliveira" (São Paulo, Brasil). Boletim Botânico da Universidade de São Paulo 17: 61-84.

Dittrich, V. A. de O., Kozera, C. \& Menezes-Silva, S. 1999. Levantamento florístico dos epífitos vasculares do Parque Barigüi, Curitiba, Paraná, Brasil. Iheringia, Série Botânica 52: 11-21.

Fontoura, T. 1995. Distribution patterns of five Bromeliaceae genera in atlantic rainforest, Rio de Janeiro State, Brazil. Selbyana 16(1): 79-93.

Fontoura, T.; Sylvestre, L. da S.; Vaz, A. M. S. \& Vieira, C. M. 1997. Epífitas vasculares, hemiepífitas e hemiparasitas da Reserva Ecológica de Macaé de Cima pp.89-101. In H. C. Lima \& R. R. GuedesBruni. Serra de Macaé de Cima: diversidade florística e conservação em Mata Atlântica. Jardim Botânico do Rio de Janeiro, Rio de Janeiro.

Freiberg, M. 1996. Spatial distribution of vascular epiphytes on three emergent canopy trees in French Guiana. Biotropica 28(3): 345-355. 
Guevara, L., Laborde, J. \& Sánchez, G. 1998. Are isolated remnant trees in pastures a fragmented canopy? Selbyana 19(1): 34-43.

Gottsberger, G. \& Morawetz, W. 1993. Development and distribution of the epiphytic flora in na Amazonian savanna in Brazil. Flora 1: 145-151.

Hietz, P. \& Hietz-Seifert, U. 1995a. Composition and ecology of vascular epiphyte communities along an altitudinal gradient in central Veracruz, Mexico. Journal of Vegetation Science 6(4): 487-498.

Hietz, P. \& Hietz-Seifert, U. 1995b. Struture and ecology of epiphyte communities of cloud forest in central Veracruz, Mexico. Journal of Vegetation Science 6(5): 719-728.

Hooper, E. A. \& Haufler, C. H. 1997. Genetic diversity and breeding system in a group of neotropical epiphytic ferns (Pleopeltis; Polypodiaceae). American Journal of Botany 84(12): 1664-1674.

IBGE. 1986. Levantamento de Recursos Naturais. v. 33. Inst. Bras. Geogr. Est., Rio de Janeiro, Brasil. 791 p.

Jarman, S. J. \& Kantvilas, G. 1995. Epiphytes on an old huon pine tree (Lagarostrobos franklinii) in Tasmanian rain-forest. New Zealand Journal of Botany 33(1): 65-78.

Johansson, D. R. 1974. Ecology of vascular epiphytes in West African rain forest. Acta Phytogeographica Suecia 59: 1-129.

Justus, J. O., Machado, M. L. A. \& Franco, M. S M. 1986. Geomorfologia. In: Levantamento de Recursos Naturais. IBGE, Rio de Janeiro, Brasil. v.33.p. 313-404.

Kersten, R. de A. \& Menezes-Silva, S. 2001. Composição florística e estrutura do componente epifítico vascular em floresta de planície litorânea na Ilha do Mel, Paraná, Brasil. Revista Brasileira de Botânica 24(2): 213-226.

Labiak, P. H. \& Prado, J. 1998. Pteridófitas epífiticas da Reserva Volta Velha, Itapoá - Santa Catarina, Brasil. Boletim do Instituto de Botânica 11: 1-79.

Madison, M. 1979. Distribution of epiphytes in a rubber plantation in Sarawak. Selbyana 5: 107-115.

Mantovani, A. 1999. Leaf morpho-physiology and distribution of epiphytic aroids along a vertical gradient in a Brazilian rain forest. Selbyana 20(2): 241-249.

Mello Filho, L. E. de \& Neves, L. de J. 1989. Anatomia foliar de Ficus organensis (Miq.) Miq. Bradea 5(15): 156-165.

Ministério do Exército. 1979. Maquiné. Região Sul do Brasil - 1:50.000, Folha SH.22-X-C-V-2 MI-297/2. (Carta cartográfica)
Mondragón, D., Durán, R., Ramírez, I. \& Olmsted, I. 1999. Population dymamics of Tillandsia brachycaulos Schltdl. (Bromeliaceae) in Dzibilchaltun National Park, Yucatán. Selbyana 20(2): 250-255.

Mursidawati, S., Norton, D. A. \& Astuti, I. P. 1999. Distribution of Pomatocalpa spicata Breda (Orchidaceae) within and among host trees in Manusela National Park, Seram, Maluku Archipelago, Indonesia. Selbyana 20(1): 116-119.

Nieder, J., Engwald, S. \& Barthlott, W. 1999. Patterns of neotropical epiphyte diversity. Selbyana 20(1): 66-75.

Nimer, E. 1979. Climatologia do Brasil. IBGESUPREN, Rio de Janeiro, Brasil. 421 p.

Nunes, V. F. \& Waechter, J. L. 1998. Florística e aspectos fitogeográficos de Orchidaceae epifíticas de um morro granítico subtropical. Pesquisas (48): 127-162.

Otero-Anaiz, A., Castillo, S., Meave, J. \& IbarraManriquez, G. 1999. Isolated pasture trees and the vegetation under their canopies in the Chiapas Coastal Plain, México. Biotropica 31(2): 243-254.

Pillar, V. de P. 1996. Multiv: Aplicativo para análise multivariada e testes de hipóteses. Depto. de Botânica, UFRGS, Porto Alegre. (Sistema eletrônico de processamento de dados).

Podani, J. 1994. Multivariate data analysis in ecology and systematics. SPB Academic Publishing BV, The Hague, Países Baixos. 316 p. ilustr.

Prosperi, J., Edelin, C. \& Michaloud, G. 1995. Modalities of aerial space occupancy by hemi-epiphytes role of shoot reiteration. Revue d'écologie - la terre et la vie 50(2): 167-187.

Putz, F. E., Romano, G. B. \& Holbrook, N. M. 1995. Comparative phenology of epiphytic and tree phase strangle fig in a Venezuelan palm savannah. Biotropica 27(2): 183-189.

Rambo, B. 1994. A fisionomia do Rio Grande do Sul. $3^{a}$ ed. Editora da Universidade do Vale do Rio dos Sinos, São Leopoldo, Brasil. 473p.

Reitz, R. 1983. Bromeliáceas e a malária-bromélia endêmica. Flora Ilustrada Catarinense (brom): $1-559$.

Rudolph, D., Rauer, G., Neider, J. \& Barthlott, W. 1998. Distributional patterns of epiphytes in the canopy and phorophyte characteristics in a western Andean rain forest in Ecuador. Selbyana 19(1): 27-33.

Senna, R. M. \& Waechter, J. L. 1997. Pteridófitas de uma floresta com araucária. I. Formas biológicas e padrões de distribuição geográfica. Iheringia, 
Série Botânica (48): 41-58.

Sevegnani, L. \& Baptista, L. R. M. 1996. Composição florística de um floresta secundária, no âmbito da Floresta Atlântica, Maquiné, RS. Sellowia 2548: 47-71.

Sillett, S. C. 1999. Tree crown struture and vascular epiphyte distribution in Sequoia sempervirens rain forest canopies. Selbyana 20(1): 76-97.

Tremblay, R.C. 1997. Distribution and dispersion patterns of individuals in nine species of Lepanthes (Orchidaceae). Biotropica 29(1): 36-45.

Tremblay, R. L., Zimmerman, J. K., Lebron, L., Bayman, P., Sastre, I., Axelrod, F. \& Alers-Garcia, J. 1998. Host specificity and low reproductive success in the rare endemic Puerto Rican orchid Lepanthes caritensis. Biological Conservancy 85(3): 297-304.

Waechter, J. L. 1985. Aspectos ecológicos da vegetação de restinga do Rio Grande do Sul, Brasil. Comunicações do Museu de Ciências PUCRS, Série Botânica (33): 49-68.

Waechter, J. L. 1992. O epifitismo vascular na Planície Costeira do Rio Grande do Sul. Tese de Doutorado. Universidade de São Carlos, São Carlos.

Waechter, J. L. 1998a. Epiphytic orchids in eastern subtropical South America, Proceedings of the 15th World Orchid Conference, Rio de Janeiro, Brasil.p. 332-341.

Waechter, J. L. 1998b. Epifitismo vascular em uma floresta de restinga do Brasil subtropical. Revista Ciência e Natura 20: 43-66.

Willians-Linera, G., Sosa, V. \& Platas, T. 1995. The fate of epiphytic orchids after fragmentation of a mexican cloud forest. Selbyana 16(1): 36-40.

Zapfack, L., Nkongmenek, A. B., Villiers, J. \& Lowman, M. 1996. The importance of pteridophytes in the epiphytic flora of some phorophytes of the Cameroonian semi-deciduous rain forest. Selbyana 17(1): 76-81.

Zimmerman, J. K. \& Olmsted, I. C. 1992. Host tree utilization by vascular epiphytes in a seasonally inundated forest (tintal) in Mexico. Biotropica 24(3): 402-407.

Zotz, G. 1998. Demography of the epiphytic orchid, Dimerandra emarginata. Journal of Tropical Ecology 14: 725-741.

Zotz, G. \& Andrade, J. L. 1998. Water relations of two co-occurring epiphytic bromeliads. Journal of Plant Physiology 152(4-5): 545-554. 